Results CFD enabled measurement of WSS throughout the thoracic aorta. WSS was significantly elevated in aortic stenosis, highest in AS-BAV(RN) (mean WSS $=37.1 \pm 4.0 \mathrm{dyn} / \mathrm{cm}^{2}$, compared to $19.9 \pm 1.9 \mathrm{dyn} / \mathrm{cm}^{2}$ for AS-BAV(RL), $25.7 \pm$ $1.2 \mathrm{dyn} / \mathrm{cm}^{2}$ in AS-TAV, $12.3 \pm 3.4 \mathrm{dyn} / \mathrm{cm}^{2}$ in AR-TAV, and $9.9 \pm 5.4 \mathrm{dyn} / \mathrm{cm}^{2}$ in healthy volunteers, $\mathrm{p}<0.05$ ). Aortic stenosis patients displayed asymmetrical WSS distributions, the greater curvature experiencing the highest WSS. OSI was lower in bicuspid right-non fusion $(\mathrm{p}<0.05)$.

Eccentricity of flow was higher in bicuspid patients (Flow $_{\mathrm{a}-}$ symmetry $=84.1 \pm 5.4 \%$, compared to $28.1 \pm 21.5$ for tricuspids, $\mathrm{p}<0.05)$. Helicity of flow was assessed by the Helical Flow Index (HFI), which was higher in bicuspid right-left fusion $\left(\mathrm{HFI}_{\text {systole }}=0.39 \pm 0.04\right.$, compared to $0.28 \pm 0.03$ for all others, $\mathrm{p}<0.05$ ).

Conclusions BAV displays eccentric flow with high helicity. Presence of AS, particularly in BAV-RN led to higher WSS and lower OSI in the greater curvature of the ascending aorta. Patient-specific CFD provides non-invasive functional assessment of the thoracic aorta, and enables development of a personalized approach to diagnosis and management of aortic disease beyond traditional guidelines.

\section{CARDIOPROTECTIVE ROLE OF HEXARELIN IN A MOUSE MODEL OF MYOCARDIAL INFARCTION}

${ }^{1}$ Hayley McDonald*, ${ }^{2}$ Jason Peart, ${ }^{1}$ Nyoman Kurniawan, ${ }^{1}$ Graham Galloway, ${ }^{3}$ Chrishan Samuel, ${ }^{1}$ Chen Chen. ${ }^{1}$ University of Queensland; ${ }^{2}$ Griffith University; ${ }^{3}$ Monash University; *Presenting Author

\subsection{6/heartjnl-2016-309890.184}

This study aimed to determine whether Hexarelin (HEX), a synthetic growth hormone secretagogue, preserves cardiac function and attenuates remodelling in mouse models of myocardial infarction.

Myocardial ischemia was induced by ligation of the left descending coronary artery in $\mathrm{C} 57 \mathrm{BL} / 6 \mathrm{~J}$ mice followed by HEX $(\mathrm{n}=16)$ or vehicle $(\mathrm{VEH})(\mathrm{n}=16)$ administration at $0.3 \mathrm{mg} / \mathrm{kg} /$ day for 21 days. Treated and Sham mice were subjected to magnetic resonance imaging using a $\mathrm{T}_{1}$-weighted late gadolinium enhancement sequence (LGE) at 9.4 Tesla (T) to measure left ventricular (LV) function, mass and infarct size at $24 \mathrm{hrs}$ and 21 days. HEX mice demonstrated a significant improvement $(\mathrm{P}<0.05)$ in ejection fraction $(\mathrm{EF})$ compared with $\mathrm{VEH}$ at $24 \mathrm{~h}(42 \%$ vs $34 \%$ respectively) and at 21 days (49\% vs $36 \%)$.

A significant decrease in LV mass, interstitial collagen and collagen concentration was demonstrated after 21 days within the HEX group. This was accompanied by a decrease in TGF-Î̃ 21 and $\hat{I} \pm$-SMA and increase in MMP-13 in the HEX group. Furthermore, heart rate variability analysis demonstrated that HEX treatment shifted the balance of autonomic nervous activity towards a parasympathetic predominance, evidenced by a smaller low/high-frequency power ratio and increased normalised high frequency power. This was combined with a significant decrease in Troponin-I, IL1- $\hat{\mathrm{I}}^{2}$ and TNF-î \pm levels with HEX treatment compared with VEH treatment after $24 \mathrm{~h}$.

These results demonstrate that GHS may preserve ventricular function and favourably remodel the process of fibrotic healing in mouse models of myocardial infarction; this may be through anti-inflammatory mechanisms.

\section{GLYCOPROTEOMICS REVEALS DECORIN PEPTIDES WITH ANTI-MYOSTATIN ACTIVITY IN HUMAN ATRIAL FIBRILLATION}

${ }^{1}$ Javier Barallobre Barreiro*, ${ }^{2}$ Shashi Gupta, ${ }^{1}$ Anna Zoccarato, ${ }^{1}$ Rika Kitazume-Taneike, ${ }^{1}$ Marika Fava, ${ }^{1}$ Xiaoke Yin, ${ }^{1}$ Anna Zampetaki, ${ }^{3}$ Alessandro Viviano, ${ }^{1}$ Mei Chong, ${ }^{4}$ Marshall Bern, ${ }^{3}$ Antonios Kourliouros, ${ }^{5}$ Nieves Domenech, ${ }^{1}$ Peter Willeit, ${ }^{1}$ Ajay M Shah, ${ }^{3}$ Marjan Jahangiri, ${ }^{6}$ Liliana Schaefer, ${ }^{7}$ Jens W Fischer, ${ }^{8}$ Renato V lozzo, ${ }^{9}$ Rosa Viner, ${ }^{2}$ Thomas Thum, ${ }^{10}$ Joerg Heineke, ${ }^{11}$ Antoine Kichler, ${ }^{1}$ Kinya Otsu, ${ }^{1}$ Manuel Mayr. ${ }^{1}$ King's College London; ${ }^{2}$ Institute for Molecular and Translational Therapeutic Strategies, $\mathrm{MH}$ Hannover; ${ }^{3}$ St Georges Hospital, NHS Trust; ${ }^{4}$ Protein Metrics; ${ }^{5}$ Biobanco a Corua, INIBICComplexo Hospitalario Universitario de A Corua; ${ }^{6}$ Klinikum Der Goethe-UniversitÃ ${ }^{7}$ Institute for Pharmacology and Clinical Pharmacology, Heinrich-Heine-University; ${ }^{8}$ Sidney Kimmel Medical College at Thomas Jefferson University; ${ }^{9}$ Thermo Fisher Scientific; ${ }^{10}$ Experimental Cardiology, Department of Cardiology and Angiology, $\mathrm{MH}$-Hannover; ${ }^{11}$ Laboratoire Vecteurs: SynthÃ"Se Et Applications ThÃ@ORapeutiques, $\hat{A}$ UMR $7199 \hat{A}$ CNRS UniversitÃ@ de Strasbourg; *Presenting Author

\subsection{6/heartjnl-2016-309890.185}

Background Myocardial fibrosis is a feature of many cardiac diseases. We used proteomics to profile glycoproteins in the human cardiac extracellular matrix (ECM).

Methods and Results Atrial specimens were analysed by mass spectrometry after extraction of ECM proteins and enrichment for glycoproteins or glycopeptides. Out of all ECM proteins identified, the small leucine-rich proteoglycan decorin was found to be the most fragmented. Within its protein core, eighteen different cleavage sites were identified. In contrast, no cleavage was observed for biglycan, the most closely related proteoglycan. Decorin processing differed between human ventricles and atria and was altered in disease. The Cterminus of decorin, important for the interaction with connective tissue growth factor, was predominantly detected in ventricles compared to atria. In contrast, atrial appendages from patients in persistent atrial fibrillation had higher levels of full-length decorin but also harboured a cleavage site that was not found in atrial appendages from patients in sinus rhythm. This cleavage site preceded the $\mathrm{N}$-terminal domain of decorin that controls muscle growth by altering the binding capacity for myostatin. Myostatin expression was decreased in atrial appendages of patients with persistent persistent atrial fibrillation and hearts of decorin null mice. A synthetic peptide corresponding to this decorin region dose-dependently inhibited the response to myostatin in cardiac myocytes and in perfused mouse hearts.

Conclusion This proteomics study is the first to analyse the human cardiac ECM. Novel processed forms of decorin protein core, uncovered in human atrial appendages can regulate the local bioavailability of anti-hypertrophic and pro-fibrotic growth factors.

\section{PLATELETS AS KEY REGULATORS OF FIBRIN-CLOT ARCHITECTURE AS ASSESSED BY FRACTAL ANALYSIS OF VISCOELASTIC PROPERTIES; EFFECTS OF STANDARD ANTI-PLATELET THERAPIES}

${ }^{1}$ Rebecca Knowles* ${ }^{2}$ Lindsay D'Silva, 1Paul-A Amstrong 1 1 Melissa Chan, ${ }^{2}$ Matthew Lawrence, ${ }^{2}$ Sophie Standford, ${ }^{2}$ Ahmed Sabre, ${ }^{1}$ Arthur Tucker, ${ }^{2}$ Phylip Williams, ${ }^{1}$ Timothy Warner, ${ }^{2}$ Phillip Evans. ' WHRI; ${ }^{2} 3$ NISCHR Haemostasis Biomedical Research Unit, ABMU Health Board; *Presenting Author

10.1136/heartjnl-2016-309890.186

Introduction Platelets are critical drivers of thrombus formation with high on-treatment platelet reactivity despite dual 